\title{
Vorticity Balance off the South Shore of Oahu, Hawaii, Derived by High-Frequency Radio Doppler Current Observations
}

\author{
Alma Carolina Castillo-Trujillo, Dale Partridge, Brian Powell, \\ AND PIERRE FLAMENT \\ Department of Oceanography, School of Ocean and Earth Science and Technology, \\ University of Hawai'i at Mānoa, Honolulu, Hawaii
}

(Manuscript received 22 December 2017, in final form 5 October 2018)

\begin{abstract}
High-frequency radio Doppler surface current observations off the south shore of Oahu, Hawaii, are used to calculate the vorticity equation at a $\sim 2-\mathrm{km}$ spatial resolution in terms of a time-dependent and time-mean surface balance. First-order terms are mean advection of mean vorticity, vortex stretching, and a residual, which is treated as unquantified terms such as wind stress curl, bottom pressure torque, and noise. The most striking feature in the 2-yr time-mean vorticity balance is the anticorrelation between advection of vorticity and vortex stretching implying that potential vorticity (PV) advection is the most dominant mechanism in the area. Several terms in the depth-integrated vorticity balance were also estimated. The bottom pressure torque acts as a first-order term only in areas of shallow topography. A PV analysis resulted in the 50-m Penguin Bank steering the westward Hawaiian Lee Current.
\end{abstract}

\section{Introduction}

Among ocean current measurements, only highfrequency radio (HFR) Doppler current meters are able to map currents at both high spatial resolution (on the order of kilometers), and temporal resolution (on the order of an hour); therefore, they can uniquely estimate the momentum and vorticity balances of the upper ocean. Estimating the momentum balance requires knowing the pressure field either through hydrography or altimetry, both of which cannot sample at the same high resolution as the HFRs. We can, however, estimate the vorticity balance without the need for the pressure gradients using only HFRs.

In this study, our objective is to investigate how the mean surface vorticity balance may be estimated and analyzed from an array of HFRs. We will first review the theoretical derivation of the surface vorticity balance and its expression in terms of measurable quantities. We then will apply the concept to an area where the vorticity forcing signal is known to be strong; the lee of the island of Oahu, Hawaii (Chavanne et al. 2010b).

The Hawaiian Islands are a prime region to study the vorticity balance since the mountainous islands

Corresponding author: Alma Carolina Castillo-Trujillo, acast@ hawaii.edu present a barrier to the trade winds generating wind stress curl (Chavanne et al. 2002), which in turn produces cyclonic and anticyclonic eddies. A complex surface circulation encompasses the islands (Fig. 1); with the North Equatorial Current (NEC) impinging to the east of them and bifurcating into the North Hawaiian Ridge Current (NHRC) to the north and the Hawaiian Lee Current (HLC) to the south of the islands.

The Hawaiian archipelago is a key region for generating mesoscale eddies in the western Pacific Ocean. A previous model study of the vorticity balance study around the Hawaiian Islands (Azevedo Correia de Souza et al. 2015) showed that wind stress curl can produce enough advection of vorticity to enhance the canonical circulation around the Hawaiian archipelago and thus modify the North Pacific Ocean circulation. South of the archipelago, Yoshida et al. (2011) found the mesoscale variability induced not only by the wind stress curl but by the instability of the sheared NEC and the Hawaiian Lee Counter Current (HLCC) flowing eastward south of the HLC.

The goal of this paper is to calculate the mean vorticity balance from 2 years of HFR surface velocities. To account for the surface frictional forcing, the vorticity balance residual will be compared to the wind stress curl from an atmospheric model in conjunction with the mixed layer depth from an ocean general circulation model (GCM). 


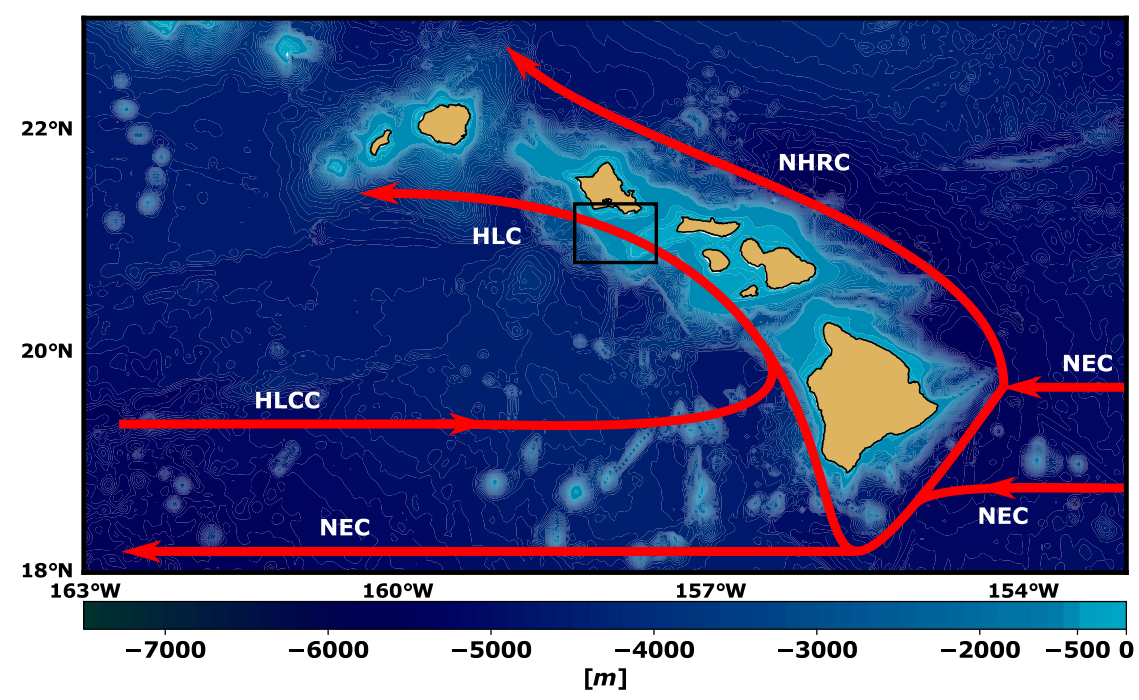

FIG. 1. Schematic map of the mean surface currents around Hawaii overlaid on the bathymetry. Labels indicate the Hawaiian Lee Current (HLC), the North Equatorial Current (NEC), the Hawaiian Lee Counter Current (HLCC), and the North Hawaiian Ridge Current (NHRC). The black square indicates the area of study.

The paper will be presented as follows: section 2 describes the theoretical background, data and methods are described in section 3, the HFR observations are described in section 4 , and the surface vorticity balance is described in section 5 . The results are discussed in section 6 and summarized in section 7 .

\section{Theoretical background}

The surface quasigeostrophic vorticity balance equation involves quantities directly measurable by the HFR. Assuming hydrostatic balance and incompressibility the horizontal momentum equation is

$$
\partial_{t} \mathbf{u}+\mathbf{u} \cdot \nabla \mathbf{u}+f \times \mathbf{u}=\frac{1}{\rho} \nabla p+\mathbf{F},
$$

in which $\mathbf{u}=[u(x, y), v(x, y)], p$ is pressure, $\rho$ is density, $\nabla$ is the two-dimensional del operator $(\partial / \partial x, \partial / \partial y)$, and F contains the forcing and dissipative terms. Taking the horizontal curl of Eq. (1) yields the surface vorticity conservation equation in which pressure effects are no longer explicit:

$$
\partial_{t} \zeta+\mathbf{u} \cdot \nabla(\zeta+f)+(\zeta+f) \nabla \cdot \mathbf{u}=\nabla \times \mathbf{F} .
$$

On the lhs of Eq. (2) the first term is the rate of change of relative vorticity; the second and third terms are the advection of relative and planetary vorticity $(\beta=d f / d y)$, respectively; and the fourth term is the vortex stretching. On the rhs $\nabla \times \mathbf{F}$ acts as the sources and sinks of vorticity caused by external forcing and dissipation.
HFRs measure currents over a thin layer of depth $H_{R}$ $\sim 0.7 \mathrm{~m}$ (at $16 \mathrm{MHz}$ ) (Stewart and Joy 1974; Gurgel et al. 1999a; Röhrs et al. 2015). While this depth is ill-defined and there are no closed theoretical expressions for it, it is much smaller than the mixed layer depth, and thus the horizontal momentum and vorticity terms in Eq. (2) will be assumed constant within $H_{R}$. Neglected terms in Eq. (2), which cannot be directly calculated by HFR observations, include vorticity tilting and lateral stress divergences arising from the curl of the vertical momentum of advection.

\section{Data and methods}

\section{a. HFR surface currents}

Two Wellen radar (WERA) HFRs (Gurgel et al. 1999a,b), with a range of $100 \mathrm{~km}$ and a range resolution of $1.5 \mathrm{~km}$, were deployed on the south shore of Oahu, Hawaii, from September 2010 to September 2012 (labeled in Fig. 2). A description of the principles of the WERA radars can be found in Gurgel et al. (1999a). The configuration of the radars (frequency of $16 \mathrm{MHz}$, bandwidth of $100 \mathrm{kHz}$ ) and the processing techniques were identical to those detailed by Chavanne et al. (2007, 2010a). The instrument setting and radar noise are discussed in appendix A.

Figure 2a shows the location of the two radars in Kalaeloa (KAL; $21^{\circ} 18^{\prime} \mathrm{N}, 158^{\circ} 5^{\prime} \mathrm{W}$ ) and Koko Head (KOK; $21^{\circ} 26^{\prime} \mathrm{N}, 157^{\circ} 42^{\prime} \mathrm{W}$ ), covering the $670-\mathrm{m}$-deep Kaiwi Channel, and the shallow 50-m-deep Penguin Bank. The 2-yr-mean surface currents are overlaid over 

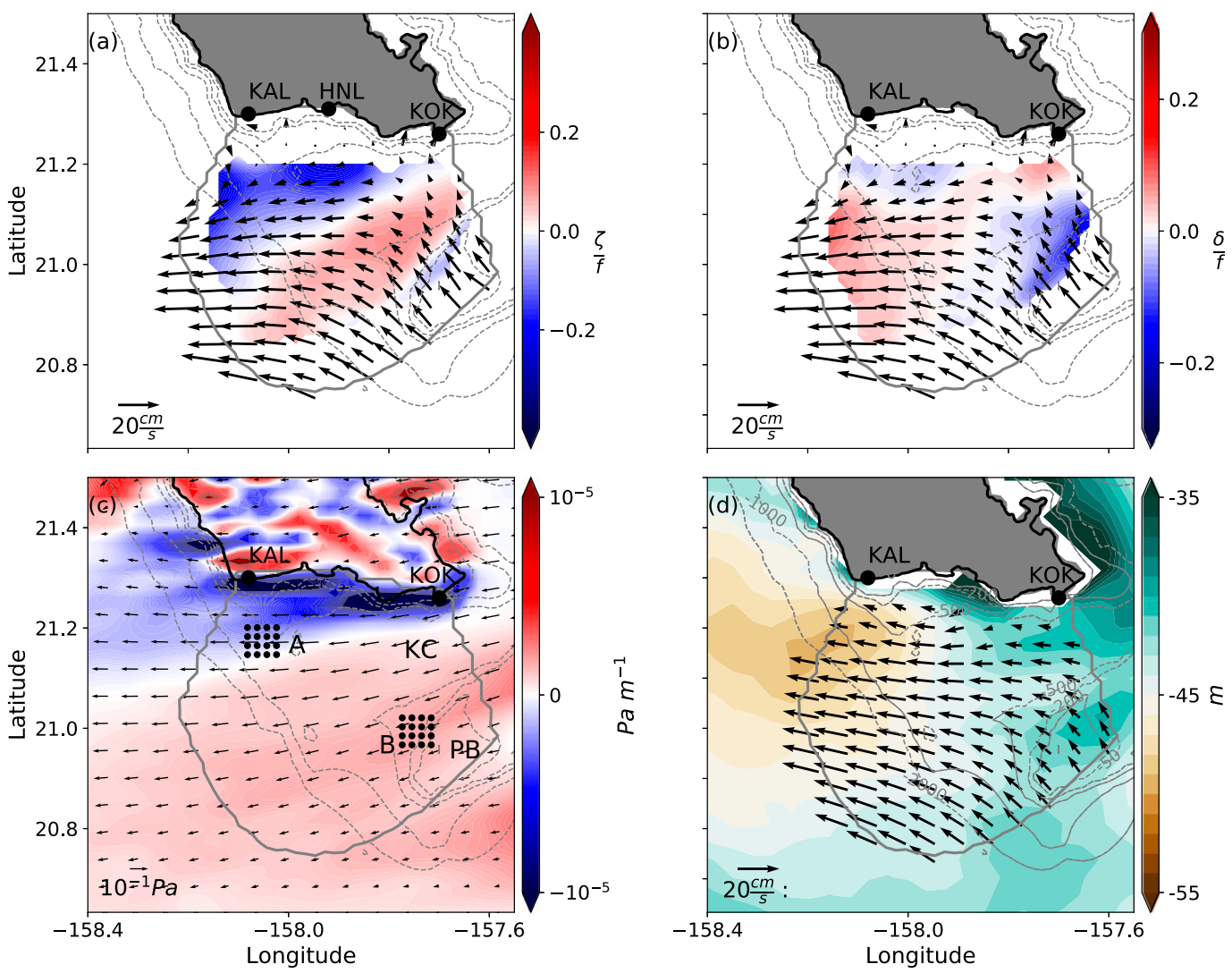

FIG. 2. (top) Time-averaged HFR surface currents overlaid on the time-averaged (a) relative vorticity and (b) divergence normalized by $f$ from $40 \%$ of the total HFR spatial coverage. (bottom) Time-averaged (c) WRF wind stress vectors overlaid on wind stress curl $\left(\mathrm{Pa} \mathrm{m}^{-1}\right)$ and (d) surface currents overlaid on the mixed layer depth (m) computed from ROMS. The Kaiwi Channel ( $\sim 600-\mathrm{m}$ depth) and Penguin Bank ( $\sim 50-\mathrm{m}$ depth) are labeled as KC and PB, respectively, in (c). All panels show the temporal mean from September 2010 to September 2012. Black dots indicate the HFR sites Kalaelola (KAL) and Koko Head (KOK) and the Honolulu airport meteorological station (HNL). Bathymetry is shown at 50,200,500, and $1000 \mathrm{~m}$. The solid gray line indicates $60 \%$ of the total HFR spatial coverage.

the color-coded mean vorticity and divergence (Figs. 2a and $2 b$ ).

Figure 3a shows the rotary power spectra of velocity averaged over grid points with more than $90 \%$ of data returned over the 2 -yr period. Similar to the results of Chavanne et al. (2010b), the slope of the continuum energy at superinertial frequencies is representative of the internal wave spectrum, with no rotary asymmetry. The diurnal and semidiurnal tides, and their harmonics, dominate the superinertial band. In contrast, clockwise energy is larger than counterclockwise in the near-inertial band extending down to $0.7 f$, the broadening of which reflects the influence of local vorticity on the inertial frequency (Kunze 1985; Weller 1982). In the subinertial band of mesoscale motions, spectral energy follows the typical slope of about $-5 / 3$ (Vallis (2006, p. 380).

Figure $3 \mathrm{~b}$ shows the corresponding power spectra of divergence $(\partial u / \partial x+\partial v / \partial y)$ and local vorticity $(\partial v / \partial x-\partial u / \partial y)$ averaged over the entire domain. In the inertial and superinertial bands, divergence and vorticity have similar magnitudes. There is no distinct near-inertial peak, since inertial motions are spatially homogeneous to first order, responding mostly to local wind forcing. Below $0.7 f$, the magnitude of vorticity progressively overwhelms divergence, as expected in flows increasing in geostrophic balance at increasingly longer periods.

Since this study is focused on the mean surface vorticity balance, temporal filtering of the HFR time series is essential to separate the mean motions from the higherfrequency oscillations. The filtering was performed in two steps. First, the tidal harmonics were removed using the t_tide algorithms (Pawlowicz et al. 2002). Second, a Butterworth low-pass infinite impulse response filter with a cutoff frequency of $0.33 \mathrm{cpd}$ (time $T=3$ days) was used to extract the subinertial mesoscale flow, thus rejecting inertial oscillations, and high-frequency waves. 

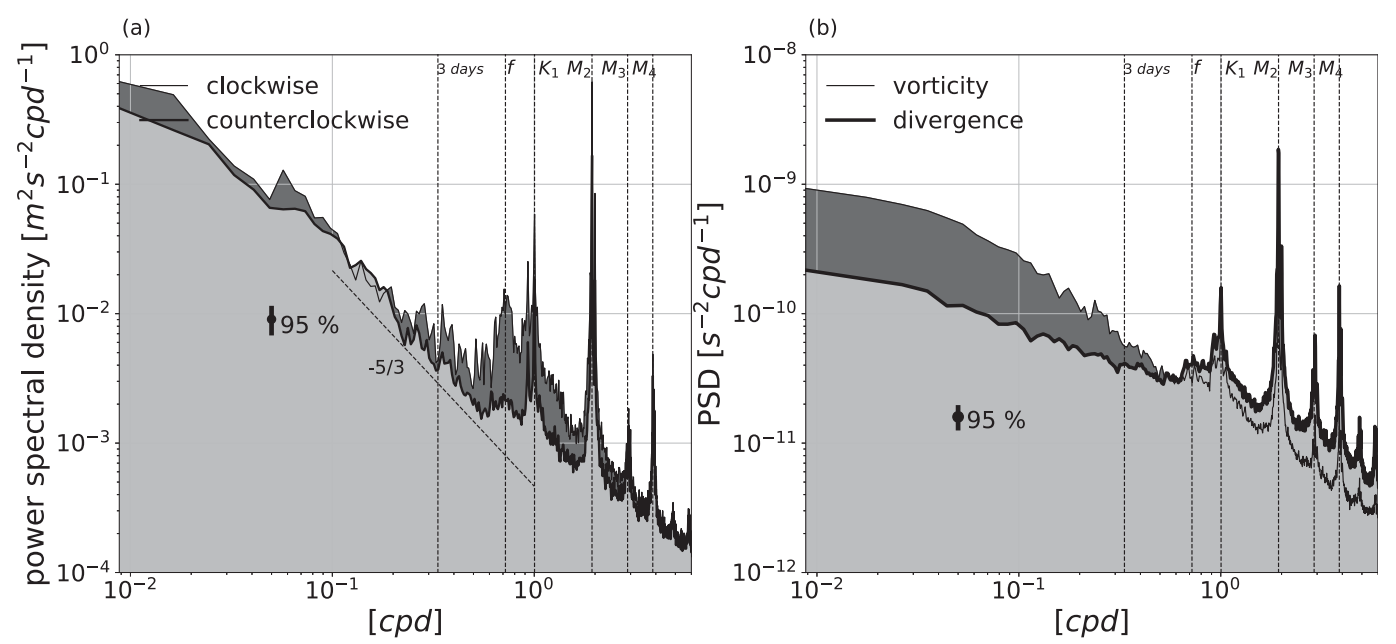

FIG. 3. (a) Rotary power spectra for September 2010-September 2012, spatially averaged over grid points with more than $90 \%$ of data returned. Clockwise and counterclockwise components are plotted with thin and thick lines, respectively. The slanted dashed line shows the $-5 / 3$ spectral slope. (b) As in (a), but for vorticity (thin line) and divergence (thick line). The $95 \%$ confidence limit is shown in both panels. Vertical dotted lines indicate the major tidal constituents, the inertial frequency $f$, and the cutoff frequency of $1 / 3$ days used in this paper to calculate the vorticity budget terms in this study.

All quantities (velocity, velocity gradients, and mean and eddy vorticity terms) were computed from these low-passed currents. The lhs of Eq. (2) can be entirely calculated from these velocity and vorticity gradients while the rhs can be estimated as a residual from the lhs of Eq. (3) or calculated explicitly from the atmospheric model and GCM product as it will be shown in section $5 \mathrm{c}$.

Surface velocity gradients are typically $O\left(10^{-8}\right) \mathrm{s}^{-1}$ while relative vorticity gradients are $O\left(10^{-12}\right) \mathrm{m}^{-1} \mathrm{~s}^{-1}$. Both velocity and vorticity gradients are highly variable throughout the HFR spatial domain. Values increase up to 10 times the typical values close to the coast and away from the HFR coverage because of the geometric dilution of precision (GDOP) (appendix A); therefore, only $40 \%$ of the total HFR spatial coverage (shown as a thin gray line in Fig. 2) is used to calculate these quantities.

\section{b. Wind measurements}

While wind stress is the primary upper-ocean forcing, the coarse resolutions of satellite winds (ASCAT, $25 \mathrm{~km}$ ) and global model winds (NCEP-NARR, $32 \mathrm{~km}$ ) do not usefully match the HFR surface velocity observations. In contrast, the network of land-based meteorological stations and ocean buoys is also too sparse to calculate gradients. Therefore, a local implementation of the Weather Research and Forecasting (WRF) Model (Tu and Chen 2011), assimilating data from satellite, aircrafts, and buoys, will be used to provide the surface wind field at spatiotemporal resolutions matching the radar grid.
The HNL airport daily wind observations (shown in Fig. 2a) and the WRF wind-model grid point closest to the HNL station are significantly correlated with an $r$ value of 0.60. WRF wind stress is computed using Large and Pond's (1981) standard methods. Figure 2c shows the 2-yr mean surface wind stress (September 2010-September 2012; same as HFR data), overlaid over the color-coded mean wind stress curl. The wind stress field shows the dominance of the northeasterly trade winds, while the wind stress curl field reveals the influence of mountainous island topography. In particular, positive wind stress curl yielding upward Ekman pumping (cyclone generating) is observed over Penguin Bank and the Kaiwi Channel, while negative wind stress curl yielding downward Ekman pumping (anticyclone generating) is observed in the southwest coast of Oahu (Chavanne et al. 2010b).

\section{c. Ocean model mixed layer depth}

In this study, we will use the mixed layer depth from an assimilative GCM based on the Regional Ocean Modeling System (ROMS). A free-surface, hydrostatic, primitive-equation model discretized with a terrainfollowing vertical coordinate system (Shchepetkin and McWilliams 2005). The assimilation system is based on previous work (Azevedo Correia de Souza et al. 2015; Janeković et al. 2013; Matthews et al. 2012; Powell et al. 2009). It uses four-dimensional variational (4D-Var) assimilation to integrate data from the radial currents of available HFRs including KAL and KOK (described in section 3a), satellites, autonomous gliders, Argo drifters, and other in situ measurements. The assimilative model 
was compared with observations in Azevedo Correia de Souza et al. (2015) and Matthews et al. (2012). They found that in most cases, the assimilation reduces the root-mean-square error between model and observations. In particular, in Matthews et al. (2012), the model mixed layer depth in the lee of the Hawaiian Islands was compared to climatology from observations, resulting in differences of less than $10 \mathrm{~m}$. Barotropic forcing is from the Oregon State University TOPEX/Poseidon Global Inverse Solution tidal model (TPXO; Egbert and Erofeeva 2002) and the lateral forcing is provided by the coarserresolution global HYCOM. The surface forcing uses the high-resolution winds from the $1.5-\mathrm{km}$ WRF atmospheric model described in section $3 b$.

The model was run to overlap with the HFRs time series from September 2010 to September 2012 at 4-km resolution over a domain covering the Hawaiian Islands $\left(17^{\circ}-24^{\circ} \mathrm{N}, 153^{\circ}-163^{\circ} \mathrm{W}\right)$, with 32 terrain-following vertical levels: 9 levels span the upper $200 \mathrm{~m}$ in deep water, with more levels near the surface as the slope ascends toward the islands. The model output provides estimates of the temperature, salinity, and currents at a 3-h temporal resolution. The mixed layer depth, most important for our objective, was estimated as the depth where density varies $0.125 \mathrm{~kg} \mathrm{~m}^{-3}$ from the surface.

Figure $2 \mathrm{~d}$ shows the spatial distribution of the 2-yr temporal mean of ROMS surface velocities and mixed layer depth $H_{s}$. Note the large spatial variations of $H_{s}$ over the domain, ranging from $\sim 35 \mathrm{~m}$ just west of Penguin Bank, to $\sim 55 \mathrm{~m}$ southwest of the coast of Oahu. These variations are, in part, the response of the ocean to the patterns of wind stress curl (Fig. 2c), through one-dimensional mixed layer deepening and Ekman transport divergence, and in part caused by the geostrophic signature of the mean currents, which are not solely locally wind driven.

\section{Description of HFRs observations}

Figure 2 a shows the time-averaged HFR surface velocities from 2010 to 2012; a westward drift is observed, intensifying southward away from the island, with typical speeds of $25 \mathrm{~cm} \mathrm{~s}^{-1}$, corresponding to the local expression of the HLC (Lumpkin 1998; Lumpkin and Flament 2013). The time-mean relative vorticity has typical values $O\left(10^{-6}\right) \mathrm{s}^{-1}$, reflecting the topography with positive (cyclonic) vorticity $\sim+0.25 f$ immediately west of Penguin Bank, and negative (anticyclonic) vorticity $\sim-0.26 f$ closer to the coast of Oahu. The $\zeta$ values estimated over the 2-yr period are slightly more negative (53\%) than positive (47\%). There is a slight preference for positive $\zeta(57 \%)$ in the Kaiwi Channel, while the opposite is true for Penguin Bank with $65 \%$ of the total $\zeta$ values being negative.
We chose two areas of approximately $20 \mathrm{~km}^{2}$ (Fig. 2c) where GDOP is low (appendix A) such that each region is representative of (i) an area of $\sim 600-\mathrm{m}$ depth with a mean negative wind stress curl (region A) and (ii) a shallow bank 200 -m depth with a mean positive wind stress curl (region B). Northeasterly trade winds are observed throughout most the year with southwest wind events in winter and spring (Figs. 4c and 4d). In general, over the 2-yr period, both regions present a westward surface flow associated to the HLC (Castillo-Trujillo 2014). In region $B$, the frequent eastward current reversals shown in Fig. $4 \mathrm{~b}$ are associated with the presence of southwesterly winds (Castillo-Trujillo 2014) while during northeast winds the surface current is mostly northwest. On the other hand in region A (Fig. 4a) periodical southwest current reversals are seen throughout the time record, which do not seem related to any wind event but were also seen in Chavanne et al. (2010b) HFR observations off the west coast of Oahu.

\section{Surface vorticity balance}

\section{a. Low-frequency time-varying balance}

The time-varying balance in region $\mathrm{A}$ is a combination of advection of vorticity, vortex stretching, and its residual (Fig. 5a), while in region B (Fig. 5b) the balance is mostly composed of vortex stretching and residual and to a lesser extent to advection of vorticity. The residual in region A occurs either because stretching and advection of vorticity are out phase when of opposite sign or because stretching mostly dominates compared to advection of vorticity. An exception is the period from March to June 2012 when advection of vorticity is larger than vortex stretching. In contrast, in region $B$ the residual occurs because the strong stretching is opposed by the residual and sometimes weak advection. The advection of planetary vorticity is an order of magnitude smaller and does not contribute to the balance in any of the areas. In both regions, typical values of advection of vorticity and vortex stretching are around $0.5 \times 10^{-9} \mathrm{~s}^{-2}$ with maximum values at $1 \times 10^{-9} \mathrm{~s}^{-2}$.

Although the tendency term $\partial \zeta / \partial t$ is two orders of magnitude smaller than the rest of the terms in Eq. (2) (Fig. 5c), variations of $\partial \zeta / \partial t$ are generated by changes in time over different leading-order terms. For example, in June 2011 (region A) advection of vorticity and vortex stretching contribute to the term while in March 2012 (region B) vortex stretching and the residual are the main contributors.

Eddy kinetic energy (EKE) and relative vorticity peaks stand out throughout the time series with events lasting $\sim 20$ days (Figs. 5d and 5e). The largest EKE events are found in spring 2012 with values of up 

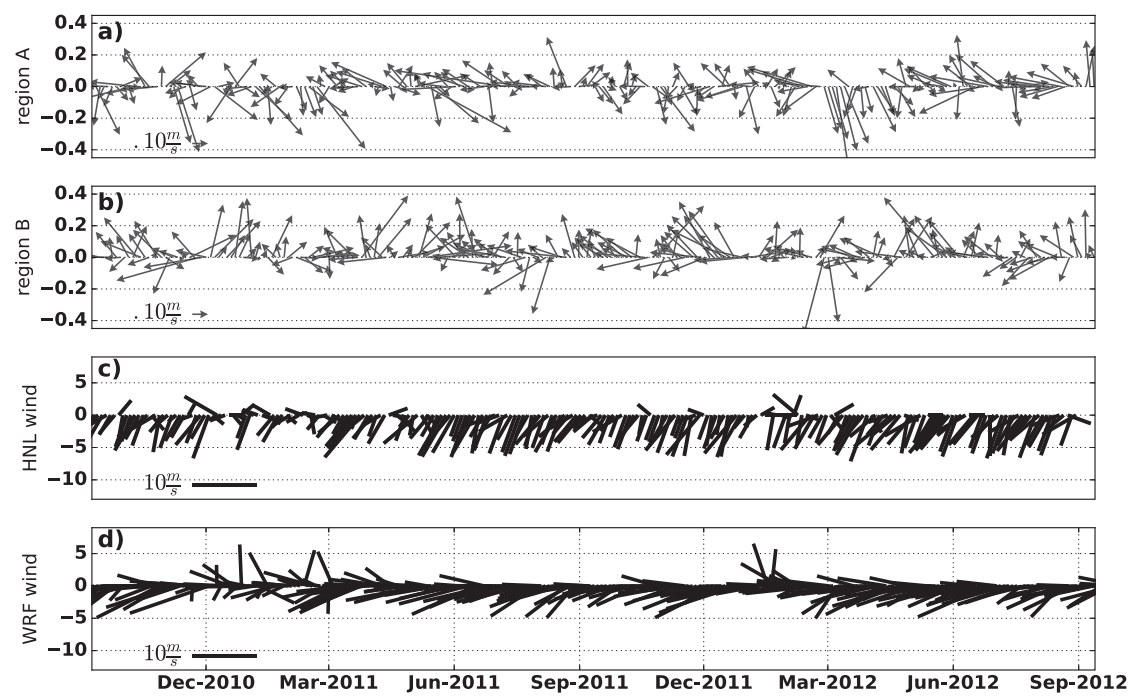

FIG. 4. Time series of spatially averaged HFR detided surface currents from (a) region A and (b) region B, defined in Fig. 2c. (c) Honolulu airport wind vectors and (d) WRF wind vectors spatially averaged over $60 \%$ of the total HFR spatial coverage.

to $\sim 0.10 \mathrm{~m}^{2} \mathrm{~s}^{-2}$. This event is also seen as an increase in positive relative vorticity with values of around $1 f$. Region A has the largest relative vorticity values reaching up to $1 f$, while region B's values are never larger than $0.5 f$. Only in region A is a slight seasonality observed in relative vorticity where large positive events are seen in winter months from December to March.

\section{b. Time-mean balance}

It is clear from the time series of EKE and relative vorticity that the HFR-derived flow is highly turbulent with relative vorticity values sometimes 5 times larger than the temporal mean (Fig. 2a). Spatial maps of the standard deviation of EKE and normalized relative vorticity are shown in Figs. 6a and 6b. The largest standard deviation values are observed in the northwest corner of the domain, while the EKE variability increases southwestward away from the coast. These large values could have an impact on the low-frequency vorticity budget. To quantify the effect of eddy vorticity forcing on the mean flow, the standard Reynolds decomposition of velocity into a time-mean and

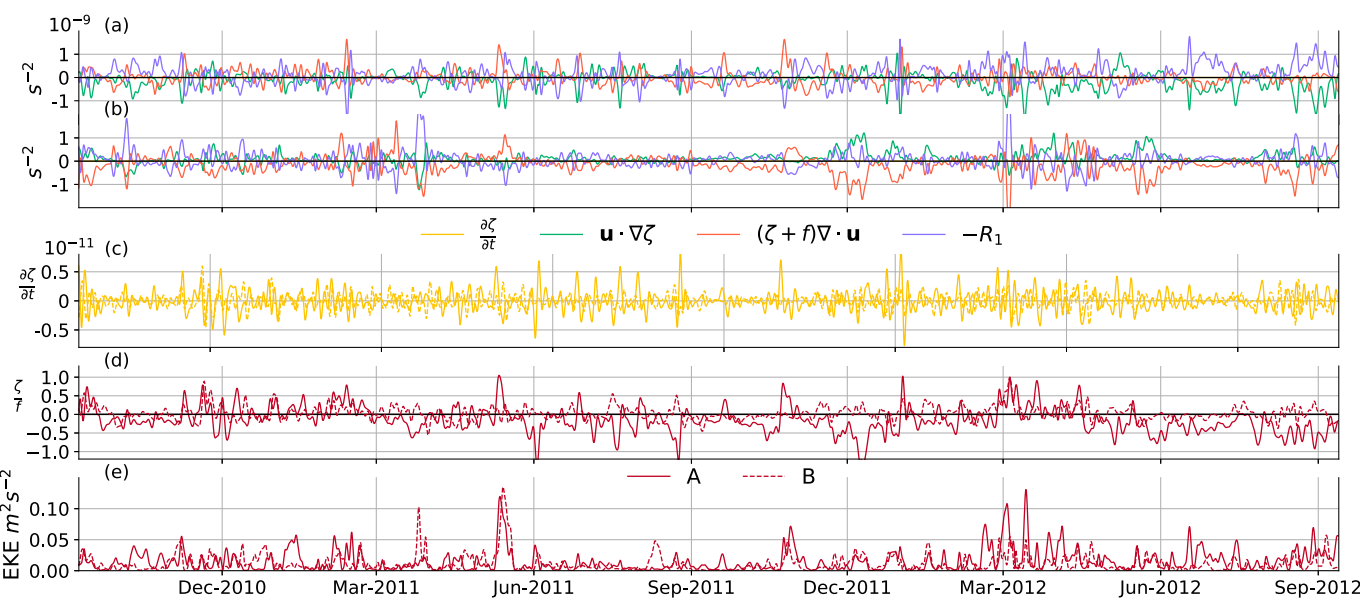

FIG. 5. Time series of spatially averaged advection of vorticity (green line), vortex stretching (orange line), and the sum of these terms defined as $R_{1}$ (purple line) for (a) region $\mathrm{A}$ and (b) region $\mathrm{B}$, units are $O\left(10^{-9}\right) \mathrm{s}^{-2}$. (c) Time series of normalized relative vorticity (red line) and change of rate of relative vorticity (yellow line) with units $O\left(10^{-11}\right) \mathrm{s}^{-2}$. (d) Eddy kinetic energy. Solid and dashed lines represent spatial average over regions A and B defined in Fig. 2c. All quantities are calculated from the 3-day low-passed HFR surface currents. 

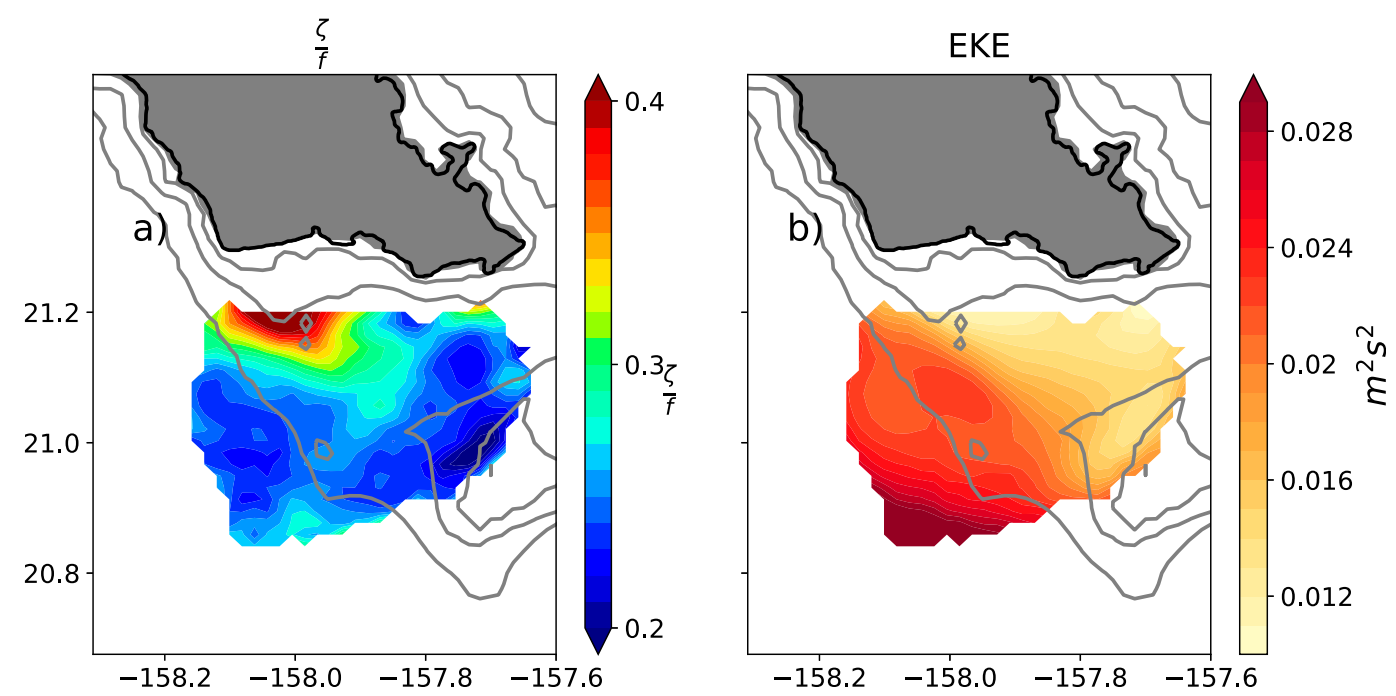

FIG. 6. Standard deviation from September 2010 to September 2012 of (a) normalized relative vorticity and (b) EKE calculated from the HFR surface velocities.

time-dependent eddy component is applied as $\mathbf{u}=\overline{\mathbf{u}}+\mathbf{u}^{\prime}$, where $\overline{\mathbf{u}}$ is the 2 -yr time mean and $\mathbf{u}^{\prime}$ is the deviation from the mean. The mean advection of mean vorticity $(\overline{\mathbf{u}} \cdot \nabla \bar{\zeta})$ and vortex stretching $[(\bar{\zeta}+f) \nabla \cdot \overline{\mathbf{u}}]$ are separated from the eddy advection of eddy vorticity $\left(\overline{\left(\mathbf{u}^{\prime} \cdot \nabla \zeta^{\prime}\right.}\right)$ and

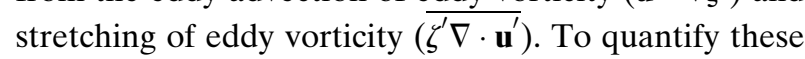
Reynolds stresses and their contribution to the mean balance they are combined as the divergence of the eddy flux of relative vorticity $\left(\nabla \cdot \overline{\mathbf{u}^{\prime} \zeta^{\prime}}\right)$. Equation (2) becomes

$$
\overline{\mathbf{u}} \cdot \nabla \bar{\zeta}+(\bar{\zeta}+f) \nabla \cdot \overline{\mathbf{u}}=\nabla \times \overline{\mathbf{F}}-\nabla \cdot \overline{\bar{u}^{\prime} \zeta^{\prime}} .
$$

Equation (3) expresses that in the absence of dissipation and mixing into the deeper layers, mean advection of relative vorticity is balanced by mean vortex stretching, divergence of the eddy flux of relative vorticity, and an external forcing. The advection of planetary vorticity and the tendency term are negligible since they are two orders of magnitude smaller than the leading-order terms.

The HFR-derived terms in Eq. (3) are plotted in Fig. 7; there is an anticorrelation between mean advection (Fig. 7a) and mean vortex stretching (Fig. 7b) of vorticity with negative (positive) values of advection of vorticity in the west (east) of the HFR domain. In certain areas, however, close to Penguin Bank and north of the Kaiwi Channel, advection does not seem capable of inducing the observed vortex stretching, indicating an external source of vorticity beside advection of potential vorticity (PV) anomalies.

The residual from $\overline{\mathbf{u}} \cdot \nabla \bar{\zeta}$ and $(\bar{\zeta}+f) \nabla \cdot \overline{\mathbf{u}}$ (denoted as $R_{1}$ in Fig. $7 \mathrm{c}$ ) resembles the vortex stretching pattern but is only about one-third of its size, except in the Kaiwi Channel and northwest of the HFR spatial domain.

Figure $7 \mathrm{~g}$ shows the divergence of the eddy flux of relative vorticity. It mostly follows the leading-order terms' pattern with positive (negative) values in the northwest (southeast) of the HFR spatial domain and its main contributor is the advection of eddy vorticity (Fig. 7e). This indicates that in addition to advection of vorticity, the eddy forcing could have caused compression of the vortex-stretching term in the northwest corner of the HFR spatial domain. This is also the area where the standard deviation of normalized relative vorticity has the largest values (Fig. 6a) suggesting highfrequency fluctuations are likely contributing to the mean vorticity budget.

\section{c. Vorticity balance integrated over the mixed layer depth}

If the dominant external forcing in the mean surface vorticity balance is the vertical turbulent flux of horizontal momentum, with wind stress $\tau$ as the surface boundary condition, the forcing term in Eq. (3) becomes $(1 / \rho) \nabla \times \overline{\tau_{z}}$. Integrating the forcing term over a time-mean mixed layer depth $H_{s}$, below which the turbulent stresses are neglected (Stern 1965; Price et al. 1986) and assuming the rest of the terms in Eq. (3) are constant yields

$$
\overline{\mathbf{u}} \cdot \nabla \bar{\zeta}+(\bar{\zeta}+f) \nabla \cdot \overline{\mathbf{u}}=\frac{1}{\rho \bar{H}_{s}} \nabla \times \overline{\tau_{s}}-\nabla \cdot \overline{\mathbf{u}^{\prime} \zeta^{\prime}},
$$

where $\boldsymbol{\tau}_{s}$ is wind stress at the surface. In Eq. (3) $\nabla \times \overline{\mathbf{F}}$ was calculated from the equation residual while in Eq. (4) it is explicitly computed from a combination of 

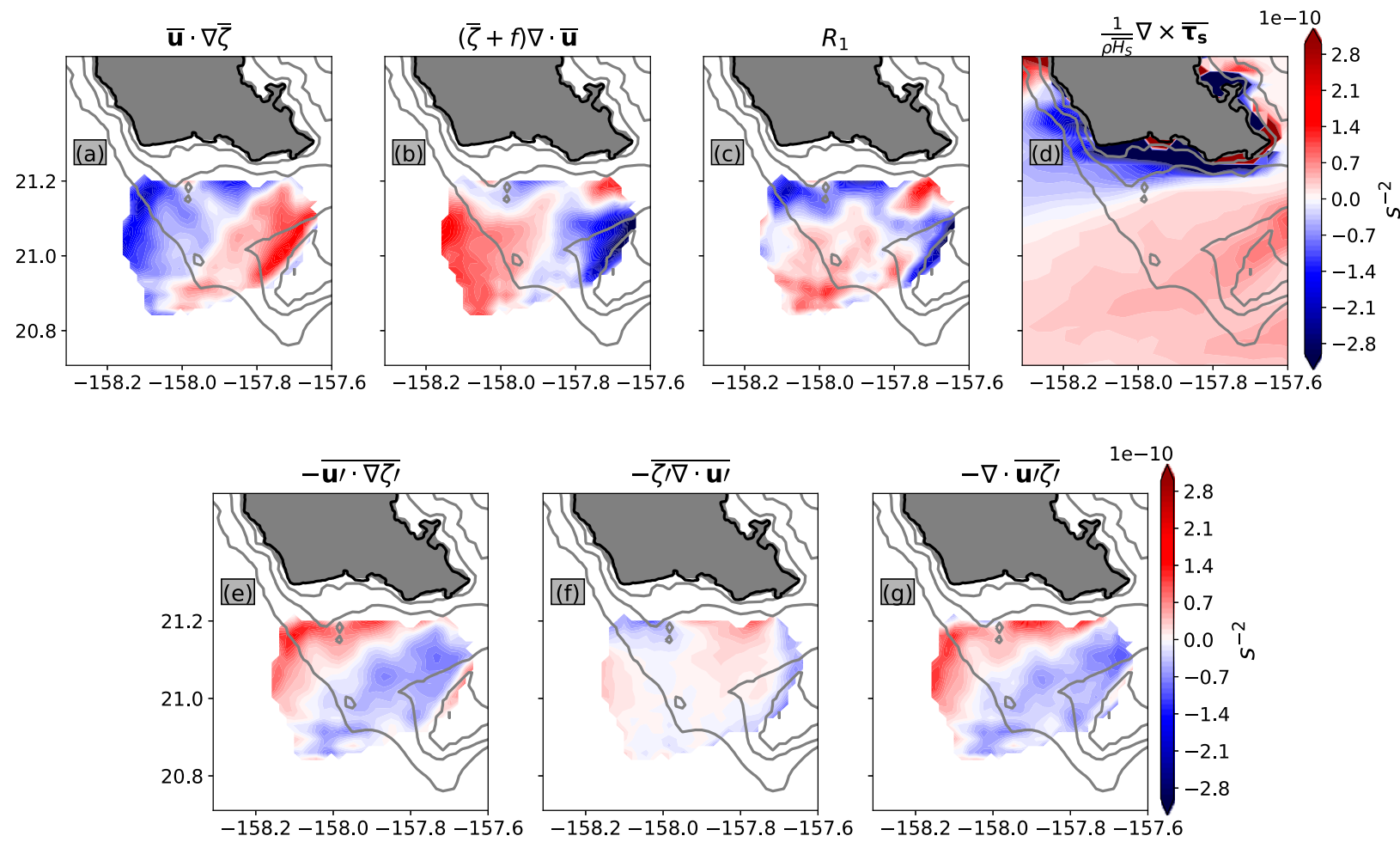

FIG. 7. Temporally averaged vorticity terms from September 2010 to September 2012 derived by (a)-(c),(e)-(g) HFR surface currents and (d) a combination of ROMS-derived surface-layer depth and WRF-derived wind stress curl. (a) Mean advection of mean vorticity $(\overline{\mathbf{u}} \cdot \nabla \bar{\zeta}),(\mathrm{b})$ vortex stretching $[(\bar{\zeta}+f) \nabla \cdot \overline{\mathbf{u}}]$, (c) residual from (a) and (b) $\left(R_{1}\right)$, (d) surface frictional forcing $\left\{\left[1 /\left(\rho H_{s}\right)\right] \nabla \times \boldsymbol{\tau}_{s}\right\},(\mathrm{e})$ eddy advection of eddy vorticity $\overline{\left(\overline{\mathbf{u}^{\prime} \cdot \nabla \zeta^{\prime}}\right)}$, (f) stretching of eddy vorticity $\left.\overline{\left(\zeta^{\prime} \nabla \cdot \mathbf{u}^{\prime}\right.}\right)$, and (g) sum of (e) and (f) $\left(\nabla \cdot \overline{\mathbf{u}^{\prime} \zeta^{\prime}}\right)$.

the mean WRF wind stress shown in Fig. 2c and the mean mixed layer depth $H_{s}$ derived by the ROMS product shown in Fig. 2d.

The first term on the rhs of Eq. (4) (Fig. 7d) shows the wind stress curl variations caused by the island shadow of the trade winds (Chavanne et al. 2002, 2010b) with positive values in most of the HFR domain, except in the northwest of the area near the coast. Despite the similarity between time-averaged surface velocities from both observations and model (Figs. 2a and 2d), it is not expected that the residual from Eq. (3) will exactly resemble the modeled surface frictional forcing term. The residual includes all of the unquantified terms and noise and the spatial and temporal resolutions of both model ( $4 \mathrm{~km}$ and $3 \mathrm{~h}$ ) and HFR observations $(1.5 \mathrm{~km}$ and $1 \mathrm{~h})$ are not the same. Nevertheless, the surface forcing term has the same order of magnitude $O\left(10^{-10}\right) \mathrm{s}^{-2}$ as advection of vorticity and vortex stretching, thus consistent with the typical values of the residual. In addition, over the Penguin Bank at $21.0^{\circ} \mathrm{N}, 157.7^{\circ} \mathrm{W}$ there is an increase in positive wind stress curl in the modeled surface frictional forcing term (Fig. 7d) that it is reflected in the positive advection of vorticity (Fig. 7a) and negative vortex stretching (Fig. 7b).
Despite the similarity between time-averaged surface velocities from both observations and model (Figs. 2a and 2d), it is not expected that the residual from Eq. (3) will exactly resemble the modeled surface frictional forcing term.

\section{Discussion}

\section{a. Surface vorticity balance}

The HFR surface vorticity budget [Eq. (2)] was estimated as a time-varying and time-mean balance revealing flow dynamics south of Oahu, Hawaii. The leading-order terms in the time-mean surface balance (Fig. 5) are mean advection of vorticity, vortex stretching, and the residual, and to a lesser extent the eddy forcing. The residual is leading order, since it includes the unquantified terms such as wind stress curl and bottom pressure torque. The time-mean balance revealed certain aspects of the surface circulation around Oahu. In most of the HFR spatial domain there was a partial balance between mean advection of mean vorticity and vortex stretching (Figs. 7a and 7b) suggesting a surface circulation caused by PV anomalies. These PV anomalies can be 


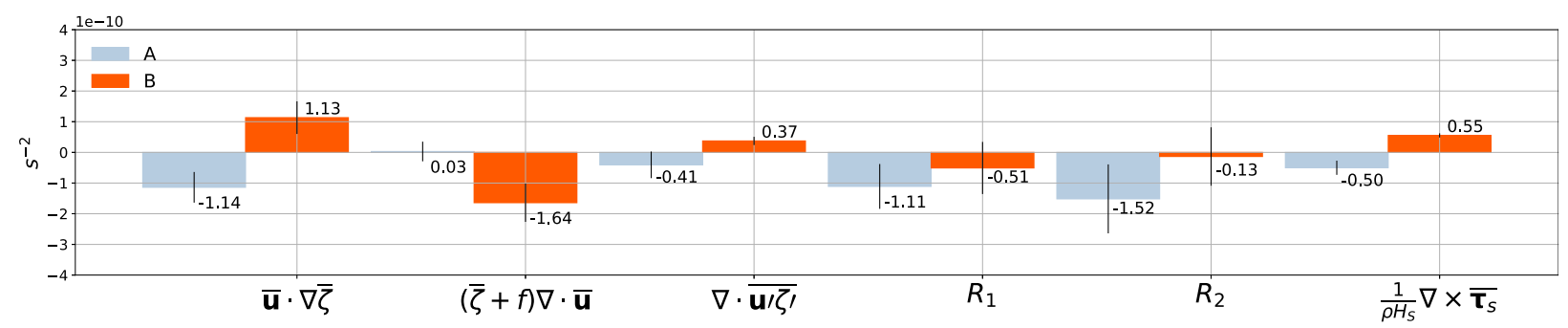

FIG. 8. Mean vorticity terms from Eq. (4) for regions A and B denoted in Fig. 2, where $R_{1}$ indicates the residual of mean advection of mean vorticity $(\overline{\mathbf{u}} \cdot \nabla \bar{\zeta})$ and vortex stretching $[(\bar{\zeta}+f) \nabla \cdot \overline{\mathbf{u}}]$ and $R_{2}$ indicates $R_{1}$ plus divergence of eddy flux of relative vorticity $\left(\nabla \cdot \overline{\mathbf{u}^{\prime} \zeta^{\prime}}\right)$. The standard deviation over each of the regions is shown in black lines.

associated to the frequent mesoscale eddies flowing westward from the island of Hawaii (Calil et al. 2008; Yoshida et al. 2011; Jia et al. 2011). In some areas, however, the mean advection of mean vorticity did not produce vortex stretching of the correct sign, such as in the northwest corner of the HFR spatial domain and north of Penguin Bank implicating forcing by bathymetry or higher-frequency eddy motions.

We will now discuss the implications of these terms in the mean vorticity budget by selecting the two areas shown in Fig. 2c. The time-mean surface vorticity balance averaged over regions $\mathrm{A}$ and $\mathrm{B}$ is summarized in Fig. 8. There are two different processes generating mean advection of mean vorticity over the HFR spatial domain since each region resulted in negative (region A) and positive (region B) mean values. Over the 2-yr record, in region $\mathrm{A}$, the advection term has a larger correlation with the residual $(r=0.79)$ compared to vortex stretching $(r=0.51)$. While in region $\mathrm{B}$, vortex stretching has a positive and larger correlation $(r=0.88)$ with the residual compared to the advection term $(r=-0.48)$. This correlation difference could be attributed to the influence of the nonmeasured HFR vorticity terms such as dissipation of stress in region $\mathrm{A}$ and the bottom pressure torque in region $\mathrm{B}$ where topography is shallow. For both regions, if the residual is compared to the frictional forcing term estimated from WRF and ROMS, values are the same of order of magnitude $O\left(10^{-10}\right) \mathrm{s}^{-2}$, even with spatial and temporal sampling differences between observations and models.

Our analysis does not include transmission of stress into the deeper layers, but in Azevedo Correia de Souza et al.'s (2015) vorticity balance, about $40 \%$ of the surface forcing is released to the deeper ocean through bottom stress. We cannot quantify this term from HFR observations, but, in region A where bathymetry is about $600 \mathrm{~m}$ it can be estimated as follows. If the residual includes only the surface frictional forcing and the transmission of stress to deeper layer, then it can be calculated as the difference between the model surface frictional forcing term $\left(-0.50 \times 10^{-10} \mathrm{~s}^{-2}\right)$ and the residual $\left(-1.11 \times 10^{-10} \mathrm{~s}^{-2}\right)$, that is, $\sim 50 \%$ of the surface forcing.

In contrast, in region $\mathrm{B}$ where bathymetry is around $200 \mathrm{~m}$, there is a positive source of mean advection of mean vorticity that balances the negative vortex stretching. Because the magnitude of the vortex stretching term is larger than the mean advection of mean vorticity, the difference could be attributed to bathymetric forcing and not to transmission of stress to bottom layers such as suggested above for region A. Thus, the residual will include not only the frictional forcing term but bottom pressure torque. This is can be quantified from Fig. 8 as follows, if we assume the total residual $R_{1}$ is $0.51 \times 10^{-10} \mathrm{~s}^{-2}$ (not including the eddy vorticity forcing) includes bottom pressure torque, we can subtract the model surface frictional forcing term $\left(0.55 \times 10^{-10} \mathrm{~s}^{-2}\right)$ from this total residual resulting in $0.04 \times 10^{-10} \mathrm{~s}^{-2}$. This closure of the vorticity budget could reveal the total residual not as surface frictional forcing but as bathymetric forcing. We will explore this further below.

The surface vorticity balance in this study does not necessarily represent the vorticity terms on a longer or larger spatiotemporal scale. Azevedo Correia de Souza et al.'s (2015) model results, although at a slightly lower spatial resolution $(4 \mathrm{~km})$, found-similar to us-that mean advection of mean vorticity and eddy vorticity forcing act as leading-order terms in the balance. In our study, the uncertainty of the eddy forcing term is as large as the residual uncertainty (appendix B). However, the inclusion of the eddy forcing term to the vorticity budget reduced the residual in region $\mathrm{B}$ but not in region A (Fig. 8). Nevertheless, it is clear that high-frequency motions affect the mean budget particularly in the northwest corner of the domain where the standard deviation of vorticity is at least 3 times larger than the rest of the of the HFR spatial domain (Fig. 6a). 


\section{b. Depth-integrated vorticity balance in Penguin Bank}

We will now further assess the influence of the bottom pressure torque term in the vorticity budget, in particular in region B where stretching of vorticity was larger than the mean advection of mean vorticity. This can be estimated assuming the vertical distribution of the mean flow around the island of Oahu is governed by a function $D(z)$, which is 1 at the surface and decays exponentially with depth as $D(z)=e^{z / H_{o}}$, where $z$ is depth measured in meters from the ocean surface, positive upward, and $H_{o}$ is the $e$-folding depth. Taking the curl of the vertically integrated equation in Eq. (2) from the surface to the seafloor using $D(z)$ and wind stress as the surface frictional forcing leads to

$$
\begin{aligned}
d_{1} \mathbf{u} \cdot \nabla \zeta+d_{o}(f+\zeta) \nabla \cdot \mathbf{u}= & \frac{1}{\rho} \nabla \times \boldsymbol{\tau}_{s}-\frac{1}{\rho} \nabla \times \boldsymbol{\tau}_{b} \\
& +J\left(P_{b}, H\right)
\end{aligned}
$$

where $d_{o}=\int_{-H}^{0} D\left(z^{\prime}\right) d z$ and $d_{1}=\int_{-H}^{0} D^{2}\left(z^{\prime}\right) d z$. The subscript $s$ refers to quantities at the surface, and $b$ to those at the seafloor. The coefficients indicate that the advection term will decay faster than vortex stretching with depth and their surface anticorrelation can be modified in the vertical as shown by Hughes (2005).

In shallow regions, the difference between the advection and vortex stretching terms will be larger than in deep regions. Table 1 shows the depth integration balance coefficients as a function of the $e$-folding depth and bathymetry. Both $d_{o}$ and $d_{1}$ increase nonlinearly with the $e$-folding depth when the depth increases. If $H_{o}$ is larger than $200 \mathrm{~m}$ and bathymetry is larger than $1000 \mathrm{~m}$, the depth-integrated vortex stretching will increase two orders of magnitude from its surface values while the depth-integrated advection of mean vorticity will increase only one order of magnitude.

Using the vertical structure fit found with ADCP observations west of Oahu by Chavanne et al. (2010b), the $e$-folding depth is $H_{o}=\sim 100 \mathrm{~m}$. In region B were ocean depth is around $200 \mathrm{~m}, d_{o}=86 \mathrm{~m}$ and $d_{1}=49 \mathrm{~m}$; therefore, the depth-integrated vortex stretching will increase $50 \%$ from the mean surface values whereas the depth-integrated mean advection of mean vorticity will increase one order of magnitude from the mean surface values. In contrast, in region A where bathymetry is around $600 \mathrm{~m}, d_{o}=100 \mathrm{~m}$ and $d_{1}=50 \mathrm{~m}$; that is, the depth-integrated vortex stretching will be $20 \%$ larger than in region B while the depth-integrated advection term will increase one order of magnitude as in region $\mathrm{B}$. Thus, both the depth-integrated advection and vortex stretching terms on the lhs of Eq. (5) will result in
TABLE 1. Depth-integrated balance coefficients assuming an equivalent barotropic flow as a function of the $e$-folding depth $H_{o}$ for various bathymetries of 50,200 , and $1000 \mathrm{~m}$.

\begin{tabular}{lccr}
\hline \multicolumn{1}{c}{$H_{o}$} & $50 \mathrm{~m}$ & $200 \mathrm{~m}$ & $1000 \mathrm{~m}$ \\
\hline$d_{o}(-50)$ & 32 & 44 & 49 \\
$d_{o}(-200)$ & 49 & 126 & 181 \\
$d_{o}(-1000)$ & 50 & 199 & 632 \\
$d_{1}(-50)$ & 22 & 39 & 48 \\
$d_{1}(-200)$ & 25 & 86 & 165 \\
$d_{1}(-1000)$ & 25 & 100 & 432 \\
\hline
\end{tabular}

$O\left(10^{-8}\right) \mathrm{s}^{-2}$. Using $\boldsymbol{\tau}_{s}$ values from WRF, the surface frictional forcing term $\left[(1 / \rho) \nabla \times \boldsymbol{\tau}_{s}\right]$ will also result in values with $O\left(10^{-8}\right) \mathrm{s}^{-2}$ as the rest of the terms in the lhs of Eq. (5).

The second term on the rhs of Eq. (5) is the vorticity produced by bottom stress $\tau_{b}$. Previous studies have shown this term to be important in shallow regions (Csanady 1978; Brink and Allen 1978) but the lack of direct observations of bottom stresses does not allow for an accurate estimation. It is important to notice that in coastal and shallow areas like Penguin Bank this term could contribute to the balance and thus be part of the residual.

The contribution of vorticity by the bottom pressure torque $J\left(P_{b}, H\right)$ to the depth-integrated vorticity balance can be estimated assuming the 2-yr-mean HFR surface velocities are geostrophic. In this case, $J\left(P_{b}, H\right)$ is equal to $f \mathbf{u}_{\mathrm{bg}} \cdot \nabla H$, where $\mathbf{u}_{\mathrm{bg}}$ is the bottom geostrophic velocity and $\nabla H$ is the spatial gradient of bathymetry. For areas shallower than the $e$-folding depth $H_{o}$, the bottom geostrophic velocity will roughly equal the HFR surface mean velocity. In areas around Penguin Bank $(\sim 50 \mathrm{~m})$ where $H<H_{o}$ and spatial variations of depth $\nabla H$ are about $1 \mathrm{~m}$ every $100 \mathrm{~m}$, the bottom pressure torque would result in values $O\left(10^{-8}\right) \mathrm{s}^{-2}$ if surface velocities are larger than $\sim 15 \mathrm{~cm} \mathrm{~s}^{-1}$. This was the case throughout the time period analyzed (Figs. 4a and 4b). This result indicates that the large vortex-stretching values seen north of Penguin Bank at around $21^{\circ} \mathrm{N}, 157.7^{\circ} \mathrm{W}$ observed in the mean surface vorticity balance (Fig. 7) could be induced in part by the bottom pressure torque and thus are part of the residual.

\section{c. Topographic steering of HFR surface flow by Penguin Bank}

As shown by the depth-integrated balance, bathymetry can influence the mean flow if spatial gradients of bathymetry are large enough such as in the Penguin Bank region. This section evaluates if the Penguin Bank steers the HFR-derived surface velocities by means of PV. 

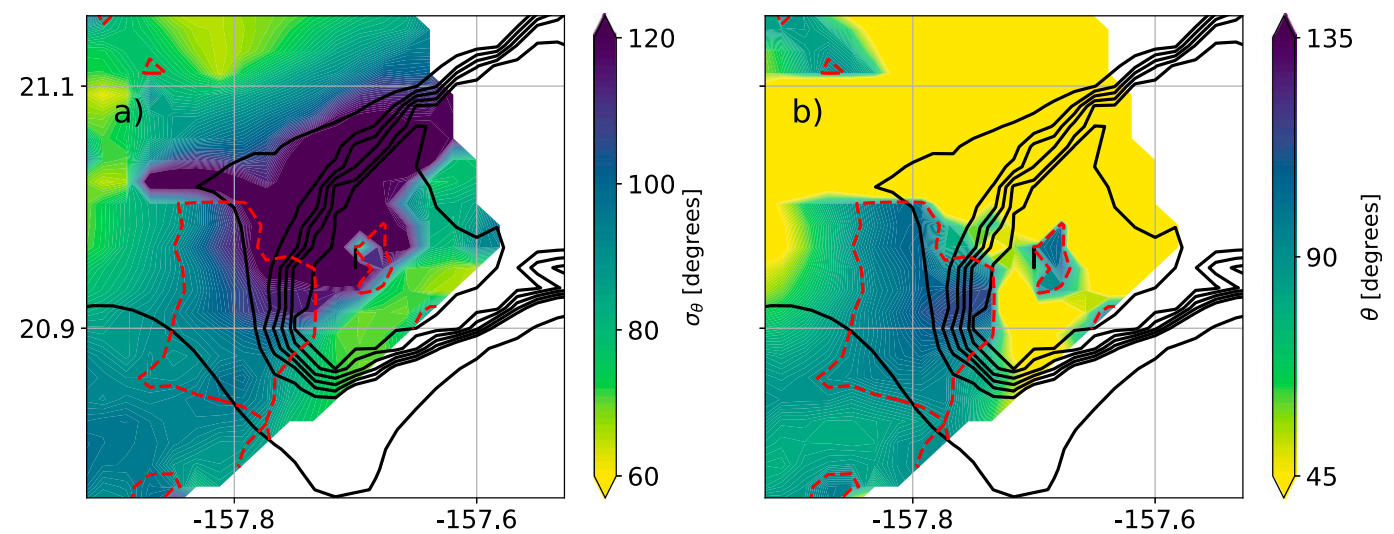

FIG. 9. (a) Standard deviation and (b) median of separation angle over the 2-yr record from September 2010 to September 2012. Separation angle is defined in degrees between the 3-day low-passed HFR surface currents and $\nabla(f / H)$. A positive angle is a clockwise angle between the surface flow and $\nabla(f / H)$. The dashed red contours indicate the areas where the median angle is $90^{\circ}$, that is, flow constrained by bathymetry assuming a barotropic flow.

Assuming a fully barotropic flow, a PV conservation can be written as $J(\psi, f / H)=0$ requiring flow to follow contours of $f / H$, where $H$ is ocean depth and $f$ is the Coriolis parameter. If $f / H$ were conserved along streamlines, then angular separation between the 3-day low-passed HFR-derived surface velocities $\mathbf{u}$ and $\nabla(f / H)$ will be $90^{\circ}$. If the angle between $\mathbf{u}$ and $\nabla(f / H)$ is mostly at right angles over the 2 -yr period with a small standard deviation, it is an indication of topographic steering of a barotropic flow (Thomson and Freeland 2003; LaCasce et al. 2008). For the area south of Oahu, this PV constrain will incite a clockwise circulation around the $\sim 50-\mathrm{m}$ Penguin Bank and a counterclockwise circulation around the $\sim 700-\mathrm{m}$ Kaiwi Channel.

Figure 9 shows the standard deviation and median of the separation angle over the 2-yr time period. The smallest standard deviation $\left(90^{\circ}\right)$ was found southwest of Penguin Bank while toward the Kaiwi Channel the standard deviation increased to $120^{\circ}$. These large standard deviation values indicate that either the low-passed surface velocities are divergent, less influenced by topography, or the assumption of a barotropic flow is not valid. As shown above, Chavanne et al. (2010b) found an $e$-folding depth of $100 \mathrm{~m}$ indicating that for shallow areas the assumption of a barotropic flow can be valid. Southwest of Penguin Bank, the standard deviation was less than $90^{\circ}$ and the median angle was found at around $90^{\circ}$ (Fig. 9b, red lines), indicating flow was mostly constrained by topography. In contrast, over Penguin Bank, standard deviation varied between $60^{\circ}$ and $120^{\circ}$ with a median angle around $45^{\circ}$. This is mostly due to flow modified by surface forcing (as shown above) and instrument noise (appendix B).

Figure 10 shows the histograms of separation angles from the area shown in Fig. 9a and the region only limited by the red dashed line. The histogram computed over the total area in Fig. 9a shows a bimodal distribution suggesting there are two distinct processes modifying the surface circulation. In contrast, in the histogram estimated only over the area within the red line in Fig. 9, most of the angles were found at around $90^{\circ}$. This indicates topography steering by Penguin Bank bathymetry is the main process modifying the surface circulation. This topographic steering can also be observed in the surface current time series (Fig. 4b), where in region B, northward surface components are frequent throughout the 2-yr time period.

\section{Summary}

HFRs observations reveal the regional dynamics south of Oahu through its mean surface velocity and vorticity structure. The 2-yr mean surface velocities exhibit a westward flow and mean Rossby number of order $0.2 f$. Maps of mean advection of mean vorticity and vortex stretching were produced at scales down to $2 \mathrm{~km}$ revealing spatial variations of vorticity due to bathymetry and wind stress curl.

The most striking feature of the mean surface vorticity balance is the anticorrelation between advection and stretching of vorticity, revealing flow driven by advection of PV anomalies. The residual from these terms was interpreted as the sum of unquantified terms and noise. Of the unquantified terms, we estimate that wind stress curl is the most significant contributor to the residual, in agreement with previous work by Chavanne et al. (2010b) and Azevedo Correia de Souza et al. (2015). The divergence of the eddy flux of relative vorticity was also estimated, resulting in values about one-third of the size of the leading-order terms but sometimes increasing 


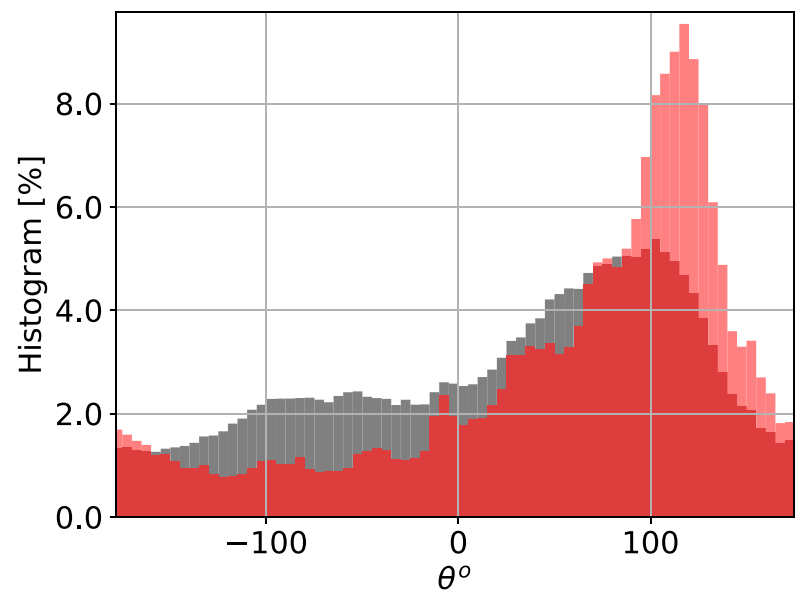

FIG. 10. Histograms of separation angle between HFR surface currents and $\nabla(f / H)$ for the region shown in Fig. 9 (black) and the region where median angle is $90^{\circ}$ in Fig. $9 \mathrm{~b}$ (red) indicating flow is constrained by bathymetry. Angles were calculated from the 3-day low-passed surface currents assuming a barotropic flow.

the first-order residual. Observations showed that the HFR-derived flow analyzed in this study is highly turbulent, with temporal fluctuations of relative vorticity sometimes 5 times larger than the temporal mean, thus likely contributing to the vorticity budget. To produce an accurate estimation of the eddy-forcing terms, the vorticity budget should be estimated with a longer HFR time series at the spatial and temporal resolution used in this study.

In shallow regions, however, bottom pressure torque accounts for most of the residual since vortex stretching is larger than advection of vorticity. An estimation of the depth-integrated vorticity equation assuming an equivalent barotropic flow was made. We found that bottom pressure torque could act a first-order term when spatial variations of topography are large enough such as the case of Penguin Bank. In addition, a PV analysis showed that bathymetry gradients can sometimes steer the mean surface circulation such as in Penguin Bank.

Time series of relative vorticity revealed intermittent episodes larger than $0.5 f$. Possible candidates to explain these large values have been observed around Hawaii in Chavanne et al. (2010b) and Azevedo Correia de Souza et al. (2015) as inertial and ageostrophic instabilities. The authors are currently working on a separate paper in which the nature of instabilities is explored with a nonassimilative model as in Gula et al. (2015). Models have shown that topographically generated instabilities $O(1 f)$ as in Gula et al. (2015) and Gula et al. (2016) are a possible pathway to energy dissipation for geostrophic flows. Therefore, it is of utmost importance to pursue and continue studies using observations measured at the submesoscale resolution.
Acknowledgments. The authors thank Matt Archer, Becky Baltes, Michael Brown, Jake Cass, Ken Constantine, Xavier Flores-Vidal, David Harris, Tyson Hilmer, Paul Lethaby, Philip Moravcik, Chris Ostrander, Charina Repollo, Ian Fernandez, and Lindsey Benjamin for assistance in the installation and maintenance of the Koko Head and Kalaeloa HFRs and João Marcos Azevedo Correia de Souza for the initial implementation of the ROMS simulations for the Hawaiian Islands. Funding for the installation and operation of the HFR is provided by the Department of Homeland Security and the Pacific Islands Ocean Observing System (PacIOOS, http:// pacioos.org), a component of NOAA's Integrated Ocean Observing System (IOOS) (Award NA11NOS0120039). This paper is School of Ocean and Earth Science and Technology Contribution Number 9546.

\section{APPENDIX A}

\section{HFR Settings and Data Processing}

The frequency-modulated continuous-wave (FCMW) HFRs were operated yielding a range resolution of 1.2$1.5 \mathrm{~km}$ and averaging times of 9-12 min yielding a time resolution of $15 \mathrm{~min}$ with each site transmitting while the other was quiet. The transmit antenna formed a beam toward the ocean, a null in the direction of the receive antennas to reduce the direct path energy. The instruments were operated in beam-forming mode with linear arrays of eight receive antennas, yielding an azimuthal resolution of $\sim 7^{\circ}$ when steering the beam normal to the receive array and degrading higher incidence angles; above $60^{\circ}$ the sidelobes are too large to obtain uncontaminated measurements (Gurgel et al. 1999b).

Vector currents were mapped on a 2-km Cartesian grid by least squares fitting the zonal and meridional component to radial measurements from both sites within a 2-km radius. A major problem is the geometric dilution of precision (GDOP), which amplifies measurement errors when the angles between the different radial directions available are closer to $0^{\circ}$ or $180^{\circ}$. Following Lipa and Barrick (1983) and Chavanne et al. (2007), we use the principal axes of the covariance matrix of the vector currents, to discard poorly constrained estimates as follows.

The currents were assumed to be constant within the search radius, where $N$ is the number of radial measurements available:

$$
m_{i}=n_{i x} u+n_{i y} v+e_{i} i=1, \ldots, N
$$

or 

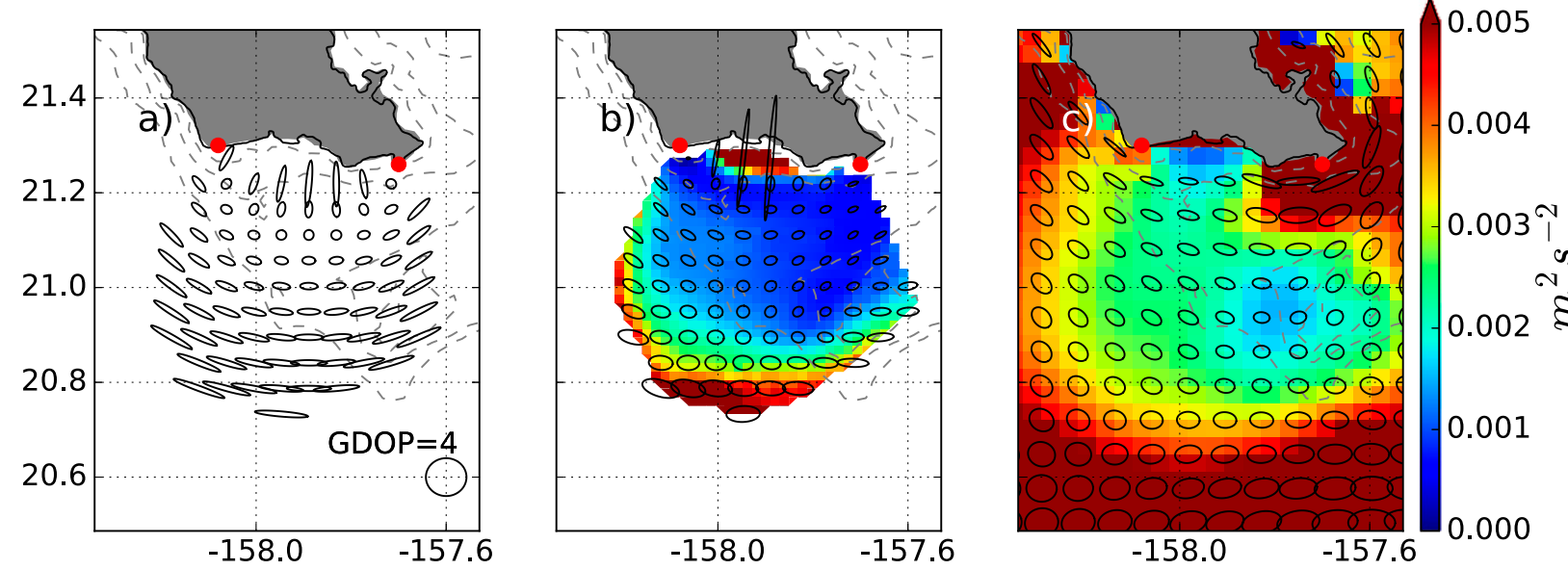

FIG. A1. (a) GDOP over the HFR domain, with GDOP $=4$ shown on the bottom-right corner. (b) Variance ellipses from the HFR surface currents and (c) from the ROMS surface currents interpolated into the HFR domain over the 2-yr time period from September 2010 to September 2012. The color bar indicates the EKE derived by HFR and ROMS variance ellipses in (b) and (c), respectively. Red dots indicate the HFR sites KAL and KOK. Bathymetry is shown as dashed gray lines at 50, 500, and $1000 \mathrm{~m}$.

$$
\mathbf{m}=\mathbf{N w}+\mathbf{e},
$$

where $\mathbf{m}$ is the vector of radial measurements, $\mathbf{N}$ is the $N \times 2$ matrix of the unit radial vectors, $\mathbf{w}=[u, v]^{\mathrm{T}}$ is the current vector, and $\mathbf{e}$ is the $N \times 1$ vector of measurements noise and model errors.

An estimate of $\mathbf{w}$ can be obtained by minimizing the sum of squared errors:
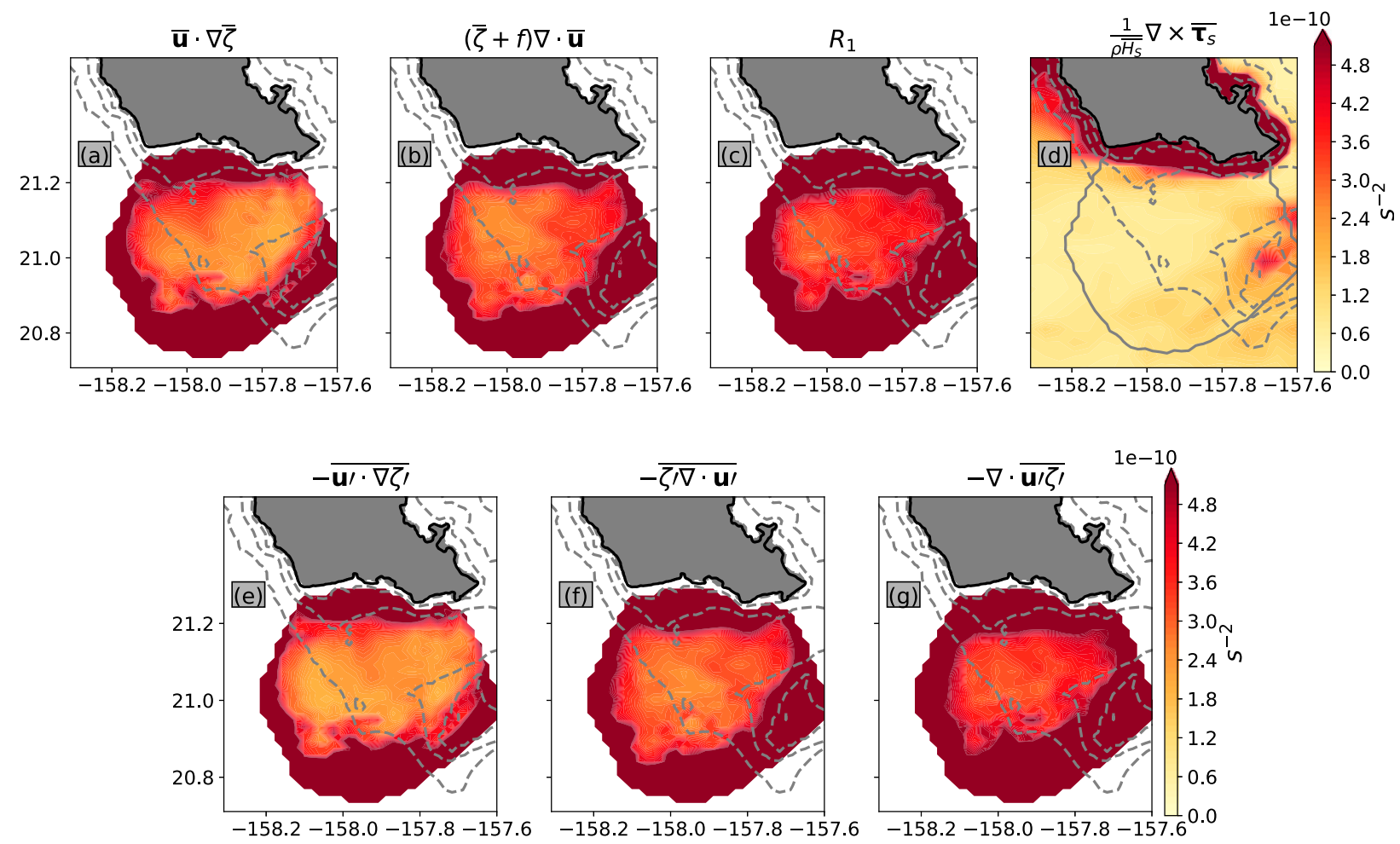

FIG. B1. Standard deviation of HFR- and model-derived vorticity terms from September 2010 to September 2012 using $60 \%$ of the total HFR spatial coverage. (a) Mean advection of mean vorticity $(\overline{\mathbf{u}} \cdot \nabla \bar{\zeta})$, (b) vortex stretching $[(\bar{\zeta}+f) \nabla \cdot \overline{\mathbf{u}}]$, (c) residual from (a) and (b) ( $\left.R_{1}\right)$, (d) surface frictional forcing $\left\{\left[1 /\left(\rho H_{s}\right)\right] \nabla \times \boldsymbol{\tau}_{s}\right\}$, (e) eddy advection of eddy vorticity $\left(\overline{\mathbf{u}^{\prime} \cdot \nabla \zeta^{\prime}}\right)$, (f) stretching of eddy vorticity $\left(\overline{\zeta^{\prime} \nabla \cdot \mathbf{u}^{\prime}}\right)$, and (g) sum of (e) and (f) $\left(\nabla \cdot \overline{\mathbf{u}^{\prime} \zeta^{\prime}}\right)$. The thin gray line in (d) denotes the $60 \%$ of the total HFR spatial coverage. The dashed gray lines denote bathymetry at 50,500 , and $1000 \mathrm{~m}$. 


$$
J=\sum_{i=1}^{N} e_{i}^{2}=\mathbf{e}^{\mathrm{T}} \mathbf{e}
$$

where the solution is

$$
\tilde{\mathbf{w}}=\left(\mathbf{N}^{\mathrm{T}} \mathbf{N}\right)^{-1} \mathbf{N}^{\mathrm{T}} \mathbf{m} .
$$

The covariance of Eq. (A4) is

$$
\mathbf{C}_{\tilde{w} \tilde{w}}=\left(\mathbf{N}^{\mathrm{T}} \mathbf{N}\right)^{-1} \mathbf{N}^{\mathrm{T}} \mathbf{C}_{e e} \mathbf{N}\left(\mathbf{N}^{\mathrm{T}} \mathbf{N}\right)^{-1},
$$

where $\mathbf{C}_{e e}$ is the covariance of $\mathbf{C}$. If the errors have the same variance and are independent of each other then

$$
\mathbf{C}_{e e}=\sigma^{2} \mathbf{I},
$$

where $\mathbf{I}$ is the unit matrix. Then the covariance of $\tilde{\mathbf{w}}$ becomes

$$
\mathbf{C}_{\tilde{w} \tilde{w}}=\sigma^{2}\left(\mathbf{N}^{\mathrm{T}} \mathbf{N}\right)^{-1} .
$$

This expression of $\sigma=1$ becomes the GDOP. Figure A1a shows the principal axes of $\mathbf{C}_{\tilde{w} \tilde{w}}$. In the present study surface velocities with GDOP greater than 4 were discarded. This high value was required since the HFR configuration increased the GDOP substantially close to the coast and away from the sites. This GDOP is seen in the HFR-derived surface current's variance ellipses and eddy kinetic energy (EKE). It is not observed in the ROMS surface currents interpolated into the HFRs since the model assimilates the HFR radial components (Figs. A1b and A1c). However, in areas where GDOP is small, in both model and observations, ellipses increase away from the coast.

\section{APPENDIX B}

\section{Vorticity Balance Errors}

The surface vorticity balance terms' standard deviations over the 2-yr time period are shown in Figure B1. Values close to the coast and away from the HFR sites where GDOP is large (appendix A) increased up to 4 times in the HFR-derived vorticity terms compared to the surface forcing term derived by WRF and ROMS. The largest values were found close to the south shore of Oahu and north of Penguin Bank. To reduce the uncertainty of the HFR-derived vorticity terms, only $40 \%$ of the total HFR spatial coverage shown in Fig. 2 as a thin gray line is used to calculate the velocity gradients and mean and eddy vorticity terms.

\section{REFERENCES}

Azevedo Correia de Souza, J. M., B. Powell, A. C. Castillo-Trujillo, and P. Flament, 2015: The vorticity balance of the ocean surface in Hawaii from a regional reanalysis. J. Phys. Oceanogr., 45, 424-440, https://doi.org/10.1175/JPO-D-14-0074.1.

Brink, K. H., and J. Allen, 1978: On the effect of bottom friction on barotropic motion over the continental shelf. J. Phys. Oceanogr., 8, 919-922, https://doi.org/10.1175/1520-0485(1978) 008<0919:OTEOBF $>2.0$. CO;2.

Calil, P. H., K. J. Richards, Y. Jia, and R. R. Bidigare, 2008: Eddy activity in the lee of the Hawaiian Islands. Deep-Sea Res. II, 55, 1179-1194, https://doi.org/10.1016/j.dsr2.2008.01.008.

Castillo-Trujillo, A., 2014: Low frequency currents south of Oahu, Hawaii. M.S. thesis, School of Ocean and Earth Science and Technology, University of Hawai'i at Mānoa, Honolulu, HI, 56 pp.

Chavanne, C., P. Flament, R. Lumpkin, B. Dousset, and A. Bentamy, 2002: Scatterometer observations of wind variations induced by oceanic islands: Implications for wind-driven ocean circulation. Can. J. Remote Sens., 28, 466-474, https://doi.org/ 10.5589/m02-047.

_- I. Janeković, P. Flament, P.-M. Poulain, M. Kuzmić, and K.-W. Gurgel, 2007: Tidal currents in the northwestern Adriatic: High-frequency radio observations and numerical model predictions. J. Geophys. Res., 112, C03S21, https://doi.org/ 10.1029/2006JC003523.

— , P. Flament, G. Carter, M. Merrifield, D. Luther, E. Zaron, and K. Gurgel, 2010a: The surface expression of semidiurnal internal tides near a strong source at Hawaii. Part I: Observations and numerical predictions. J. Phys. Oceanogr., 40, 1155-1179, https://doi.org/10.1175/2010JPO4222.1.

$\longrightarrow, \ldots$, and K.-W. Gurgel, 2010b: Interactions between a submesoscale anticyclonic vortex and a front. J. Phys. Oceanogr., 40, 1802-1818, https://doi.org/10.1175/2010JPO4055.1.

Csanady, G., 1978: The arrested topographic wave. J. Phys. Oceanogr., 8, 47-62, https://doi.org/10.1175/1520-0485(1978) $008<0047$ :TATW $>2.0$. CO;2.

Egbert, G. D., and S. Y. Erofeeva, 2002: Efficient inverse modeling of barotropic ocean tides. J. Atmos. Oceanic Technol., 19, 183-204, https://doi.org/10.1175/1520-0426(2002)019<0183: EIMOBO $>2.0 . \mathrm{CO} ; 2$.

Gula, J., M. Molemaker, and J. McWilliams, 2015: Topographic vorticity generation, submesoscale instability and vortex street formation in the Gulf Stream. Geophys. Res. Lett., 42, 4054 4062, https://doi.org/10.1002/2015GL063731.

— M. J. Molemaker, and J. C. McWilliams, 2016: Topographic generation of submesoscale centrifugal instability and energy dissipation. Nat. Commun., 7, 12811, https://doi.org/10.1038/ ncomms12811.

Gurgel, K.-W., G. Antonischki, H.-H. Essen, and T. Schlick, 1999a: Wellen radar (WERA): A new ground-wave HF radar for ocean remote sensing. Coastal Eng., 37, 219-234, https:// doi.org/10.1016/S0378-3839(99)00027-7.

-, H.-H. Essen, and S. Kingsley, 1999b: High-frequency radars: Physical limitations and recent developments. Coastal Eng., 37, 201-218, https://doi.org/10.1016/S0378-3839(99)00026-5.

Hughes, C. W., 2005: Nonlinear vorticity balance of the Antarctic Circumpolar Current. J. Geophys. Res., 110, C11008, https:// doi.org/10.1029/2004JC002753.

Janeković, I., B. Powell, D. Matthews, M. McManus, and J. Sevadjian, 2013: 4D-Var data assimilation in a nested, coastal ocean model: A Hawaiian case study. J. Geophys. Res. Oceans, 118, 5022-5035, https://doi.org/10.1002/jgrc.20389. 
Jia, Y., P. Calil, E. Chassignet, E. Metzger, J. Potemra, K. Richards, and A. J. Wallcraft, 2011: Generation of mesoscale eddies in the lee of the Hawaiian Islands. J. Geophys. Res., 116, C11009, https://doi.org/10.1029/2011JC007305.

Kunze, E., 1985: Near-inertial wave propagation in geostrophic shear. J. Phys. Oceanogr., 15, 544-565, https://doi.org/10.1175/ 1520-0485(1985)015<0544:NIWPIG > 2.0.CO;2.

LaCasce, J., O. Nøst, and P. Isachsen, 2008: Asymmetry of free circulations in closed ocean gyres. J. Phys. Oceanogr., 38, 517 526, https://doi.org/10.1175/2007JPO3789.1.

Large, W., and S. Pond, 1981: Open ocean momentum flux measurements in moderate to strong winds. J. Phys. Oceanogr., 11, 324-336, https://doi.org/10.1175/1520-0485(1981)011<0324: OOMFMI $>2.0 . \mathrm{CO} ; 2$.

Lipa, B., and D. Barrick, 1983: Least-squares methods for the extraction of surface currents from CODAR crossed-loop data: Application at ARSLOE. IEEE J. Oceanic Eng., 8, 226-253, https://doi.org/10.1109/JOE.1983.1145578.

Lumpkin, C., 1998: Eddies and currents of the Hawaiian Islands. Ph.D. thesis, School of Ocean and Earth Science and Technology, University of Hawai'i at Mānoa, Honolulu, HI, $282 \mathrm{pp}$.

Lumpkin, R., and P. J. Flament, 2013: Extent and energetics of the Hawaiian lee countercurrent. Oceanography, 26, 58-65, https://doi.org/10.5670/oceanog.2013.05.

Matthews, D., B. Powell, and I. Janeković, 2012: Analysis of fourdimensional variational state estimation of the Hawaiian waters. J. Geophys. Res., 117, C03013 https://doi.org/10.1029/ 2011JC007575.

Pawlowicz, R., B. Beardsley, and S. Lentz, 2002: Classical tidal harmonic analysis including error estimates in MATLAB using T_TIDE. Comput. Geosci., 28, 929-937, https://doi.org/ 10.1016/S0098-3004(02)00013-4.

Powell, B., A. Moore, H. Arango, E. Di Lorenzo, R. Milliff, and R. Leben, 2009: Near real-time ocean circulation assimilation and prediction in the Intra-Americas Sea with ROMS. Dyn. Atmos. Oceans, 48, 46-68, https://doi.org/10.1016/ j.dynatmoce.2009.04.001.
Price, J. F., R. A. Weller, and R. Pinkel, 1986: Diurnal cycling: Observations and models of the upper ocean response to diurnal heating, cooling, and wind mixing. J. Geophys. Res., 91, 8411-8427, https://doi.org/10.1029/JC091iC07p08411.

Röhrs, J., A. K. Sperrevik, K. H. Christensen, G. Broström, and $\varnothing$. Breivik, 2015: Comparison of HF radar measurements with Eulerian and Lagrangian surface currents. Ocean Dyn., 65, 679-690, https://doi.org/10.1007/s10236-015-0828-8.

Shchepetkin, A. F., and J. C. McWilliams, 2005: The regional oceanic modeling system (ROMS): A split-explicit, free-surface, topography-following-coordinate oceanic model. Ocean Modell., 9, 347-404, https://doi.org/10.1016/j.ocemod.2004.08.002.

Stern, M. E., 1965: Interaction of a uniform wind stress with a geostrophic vortex. Deep-Sea Res., 12, 355-367, https:// doi.org/10.1016/0011-7471(65)90007-0.

Stewart, R. H., and J. W. Joy, 1974: HF radio measurements of surface currents. Deep- Sea Res., 21, 1039-1049, https:// doi.org/10.1016/0011-7471(74)90066-7.

Thomson, R. E., and H. J. Freeland, 2003: Topographic steering of a mid-depth drifter in an eddy-like circulation region south and east of the Hawaiian Ridge. J. Geophys. Res., 108, 3341, https://doi.org/10.1029/2002JC001715.

Tu, C.-C., and Y.-L. Chen, 2011: Favorable conditions for the development of a heavy rainfall event over Oahu during the 2006 wet period. Wea. Forecasting, 26, 280-300, https://doi.org/ 10.1175/2010WAF2222449.1.

Vallis, G. K., 2006: Atmospheric and Oceanic Fluid Dynamics: Fundamentals and Large-Scale Circulation. Cambridge University Press, 745 pp.

Weller, R. A., 1982: The relation of near-inertial motions observed in the mixed layer during the JASIN (1978) experiment to the local wind stress and to the quasi-geostrophic flow field. J. Phys. Oceanogr., 12, 1122-1136, https://doi.org/10.1175/ 1520-0485(1982)012<1122:TRONIM>2.0.CO;2.

Yoshida, S., B. Qiu, and P. Hacker, 2011: Low-frequency eddy modulations in the Hawaiian Lee Countercurrent: Observations and connection to the Pacific Decadal Oscillation. J. Geophys. Res., 116, C12009, https://doi.org/10.1029/2011JC007286. 